\title{
Electron velocity distribution function in a plasma with temperature gradient and in the presence of suprathermal electrons: application to incoherent-scatter plasma lines
}

\author{
P. Guio ${ }^{1}$, J. Lilensten ${ }^{2}$, W. Kofman ${ }^{2}$, N. Bjørnå ${ }^{1}$ \\ ${ }^{1}$ The Auroral Observatory, University of Tromsø, N-9037 Tromsø, Norway, Fax: + 47776462 80; e-mail: patrick@phys.uit.no \\ ${ }^{2}$ CEPHAG Domaine Universitaire, BP 46, F-38402 St-Martin-D’hères, France
}

Received: 6 January 1998 / Revised: 13 March 1998 / Accepted: 19 March 1998

\begin{abstract}
The plasma dispersion function and the reduced velocity distribution function are calculated numerically for any arbitrary velocity distribution function with cylindrical symmetry along the magnetic field. The electron velocity distribution is separated into two distributions representing the distribution of the ambient electrons and the suprathermal electrons. The velocity distribution function of the ambient electrons is modelled by a near-Maxwellian distribution function in presence of a temperature gradient and a potential electric field. The velocity distribution function of the suprathermal electrons is derived from a numerical model of the angular energy flux spectrum obtained by solving the transport equation of electrons. The numerical method used to calculate the plasma dispersion function and the reduced velocity distribution is described. The numerical code is used with simulated data to evaluate the Doppler frequency asymmetry between the up- and downshifted plasma lines of the incoherentscatter plasma lines at different wave vectors. It is shown that the observed Doppler asymmetry is more dependent on deviation from the Maxwellian through the thermal part for high-frequency radars, while for lowfrequency radars the Doppler asymmetry depends more on the presence of a suprathermal population. It is also seen that the full evaluation of the plasma dispersion function gives larger Doppler asymmetry than the heat flow approximation for Langmuir waves with phase velocity about three to six times the mean thermal velocity. For such waves the moment expansion of the dispersion function is not fully valid and the full calculation of the dispersion function is needed.
\end{abstract}

Key words. Non-Maxwellian electron velocity distribution - Incoherent scatter plasma lines - EISCAT . Dielectric response function

Correspondence to: P. Guio

\section{Introduction}

We want to estimate the field-aligned electron mean drift velocity $V_{e}$ from incoherent scatter Doppler measurement of the plasma lines (Vidal-Madjar et al., 1975; Bauer et al., 1976; Showen, 1979). In order to do this we need to solve accurately the plasma dispersion relation for electrostatic waves at high frequencies and thus to have an accurate model of the electron velocity distribution function.

A common way of representing the whole electron velocity distribution function is to separate it into two populations: the ambient or bulk population $f_{a}(\boldsymbol{v})$ and the suprathermal or tail population $f_{s}(\boldsymbol{v})$, and special care needs to be taken for the treatment of the transition region between the suprathermal and ambient electrons. At ionospheric heights about the $F 2$ region, the bulk population of the electrons is collision-dominated and thus the velocity-space distribution is expected to be very close to a Maxwellian. In this case, the parameters describing the state of the thermal population are: the electron density $n_{e}$, the electron temperature $T_{e}$ and the potential source of inhomogeneity such as the spatial gradients of electron temperature $\nabla T_{e}$ and pressure $\nabla p_{e}$, as well as possibly an electric field $\boldsymbol{E}$. These parameters are provided by the analysis of the measurement of the ion line incoherent scattering. On the other hand, the suprathermal component $f_{s}(\boldsymbol{v})$ is taken from a complete kinetic electron transport code which takes into account the ionization and heating resulting from both solar insolation and particle precipitations.

In the first part, we describe and review the original theory developed to calculate the velocity distribution function of the ambient electrons in the presence of a temperature gradient and/or an electric field (Spitzer and Härm, 1953). Thereafter we present and discuss the calculations we use to represent the suprathermal part of the distribution function. We then describe a numerical method to calculate the full two-dimensional 
dispersion relation. We test our numerical code and discuss the results on simulated Doppler asymmetry data for radars with different wave vector and compare the results given by the heat flow approximation of Kofman et al. (1993).

\section{The ambient velocity distribution}

For low energy and for a fully ionized plasma consisting of electrons and one ion species, the distribution function of the electrons in a highly collisional regime, i.e. in a regime where the velocity-space distribution of the electrons is close to a Maxwellian (Gombosi and Rasmussen, 1991), can be approximated by the SpitzerHärm distribution function of Cohen et al. (1950) and Spitzer and Härm (1953).

This time-independent distribution function is the result of the presence of a weak electric field and a temperature gradient. The distribution function is expanded as a power series in the Knudsen number $\epsilon$ which represents the ratio of the microscopic length scale to the macroscopic length scale. In this theory only the first order in $\epsilon$ is kept, which is known as the principle of local action (Woods, 1993). This restriction to small values of $\epsilon$ implies that the electron mean free path $\lambda_{e}$ is much smaller than the different scale lengths considered $\nabla \log T_{e}, \nabla \log p_{e}$ and $e E / K_{b} T_{e}$ (Ljepojevic and MacNeice, 1989). The two Knudsen numbers associated are respectively $\epsilon_{E}$ and $\epsilon_{T}$ defined as

$\epsilon_{E}=\lambda_{e}\left(\frac{e E}{K_{b} T_{e}}-\frac{\nabla p_{e}}{p_{e}}\right)$

and

$\epsilon_{T}=2 \lambda_{e} \frac{\nabla T_{e}}{T_{e}}$,

where $E$ is the electric field, $T_{e}$ the electron temperature, $p_{e}$ the electron pressure and $\nabla$ represents the derivative along the line of sight. For small Knudsen numbers, i.e. $\epsilon_{E} \ll 1$ and $\epsilon_{T} \ll 1$, perturbation methods apply and the ambient electron velocity distribution function $f_{a}$ is expanded about a local Maxwellian $f_{0}(v)=n_{e} /$ $(2 \pi)^{3 / 2} / v_{e}^{3} \exp \left(-\left(v / v_{e}\right)^{2} / 2\right)$ with thermal velocity $v_{e}=$ $\left(K_{b} T_{e} / m_{e}\right)^{1 / 2}$ and takes the following form

$$
\begin{aligned}
f_{a}\left(x v_{e}, \mu\right)= & f_{0}\left(x v_{e}\right) \\
& {\left[1+Z \mu\left(\epsilon_{E} X_{E}(x / \sqrt{2})+\epsilon_{T} X_{T}(x / \sqrt{2})\right)\right], }
\end{aligned}
$$

where $\mu$ is the cosine of the pitch angle measured from an axis parallel to the direction of the temperature gradient and electric field, $Z$ is the charge number of the ion species and $x$ is the ratio $v / v_{e}$. The functions $X_{E}$ and $X_{T}$ are the solutions of two second-order differential equations [Eq. (40) of Spitzer and Härm (1953) and Eqs. (6)-(13) of Cohen et al. (1950)] derived from the Boltzmann's equation where only the long-range electron-electron and the electron-ion interactions have been taken into account through two Fokker-Planck collision operators. This approximation is valid for low energy only, so that the upper boundary of integration of these functions should not be too large compared to the mean thermal velocity $v_{e}$. We have recalculated the solutions to these equations for different values of the upper boundary. Figure 1 shows the two functions $X_{E}$ and $X_{T}$ for those different values of the upper boundary of integration $x_{\max }$.

By taking the first- and third-order velocity moments of the perturbation functions $X_{E}$ and $X_{T}$ one defines four transport coefficients $\gamma_{E}, \delta_{E}, \gamma_{T}$ and $\delta_{T}$. These are the normalized transport coefficients relative to a Lorentzian gas (Spitzer and Härm, 1953; Shkarofsky, 1961). Equations 4-7 show the relations between these coefficients, the velocity moments of the distribution function and the transport coefficients $\sigma_{e}, \tau_{e}, \mu_{e}$ and $\kappa_{e}$.

$$
\begin{aligned}
& \gamma_{E}=\frac{1}{3} I_{3}\left(X_{E}\right)=\frac{\sqrt{\pi} m_{e} v_{e}}{4 \sqrt{2} Z e^{2} n_{e} \lambda_{e}} \sigma_{e}, \\
& \delta_{E}=\frac{1}{12} I_{5}\left(X_{E}\right)=\frac{\sqrt{\pi} m_{e} v_{e}}{6 \sqrt{2} Z e n_{e} K_{b} \lambda_{e}} \tau_{e},
\end{aligned}
$$
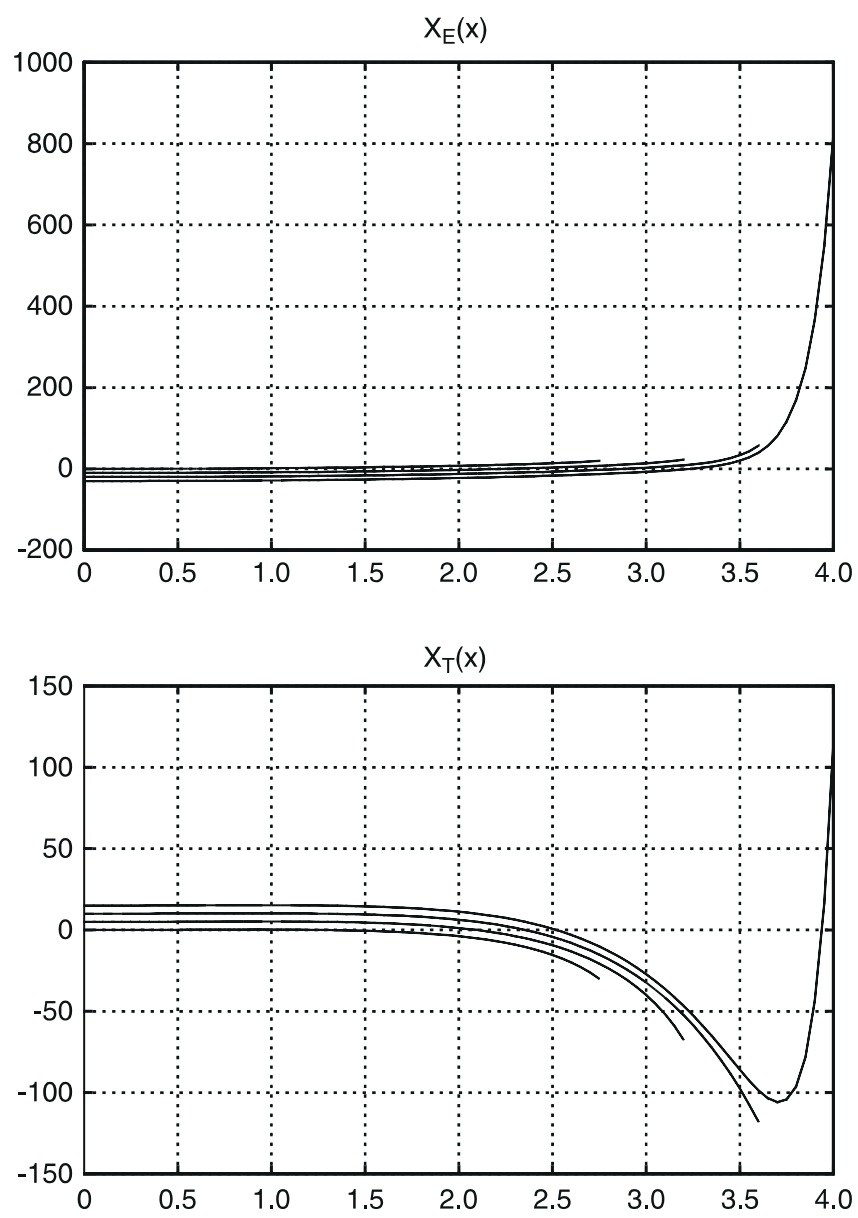

Fig. 1. The perturbation functions $X_{E}$ and $X_{T}$ integrated to different upper boundary $x_{\max }=v / \sqrt{2} v_{e}=2.8,3.2,3.6$ and 4.0, and for an ion charge number $Z=1$. Note that $X_{E}(0)=X_{T}(0)=0$ and that the $X_{E}$ 's are shifted by -10 with each other, the $X_{T}$ 's are shifted by +5 with each other, the reference curves (i.e. not shifted) are for $x_{\max }=2.8$ 
$\gamma_{T}=-\frac{4}{9} I_{3}\left(X_{T}\right)=\frac{3 \sqrt{\pi} v_{e}}{16 \sqrt{2} Z e n_{e} \lambda_{e}} \mu_{e}$

$\delta_{T}=-\frac{1}{15} I_{5}\left(X_{T}\right)=\frac{\sqrt{\pi} v_{e}}{40 \sqrt{2} Z n_{e} K_{b} \lambda_{e}} \kappa_{e}$,

with

$I_{n}(f)=\int_{0}^{x_{\max }} y^{n} f(y) \exp \left(-y^{2}\right) d y$,

where $\sigma_{e}$ is the electrical conductivity, $\tau_{e}$ is the current flow conductivity due to a temperature gradient at constant electron density, $\mu_{e}$ is the heat flow conductivity due to an electric field at constant electron temperature and $\kappa_{e}$ is the thermal conductivity.

Table 1 presents the values of the normalized transport coefficients we have recalculated and the original values of Spitzer and Härm (1953). With the exception of the values for $x_{\max }=2.8$, the values of the transport coefficients are in good agreement (under 1\%) with the values calculated by Spitzer and Härm $\left(x_{\max }=3.2\right)$.

In the work of Spitzer and Härm, the electron mean free path $\lambda_{e}$ is taken to be the mean free path due to electron-electron collisions and electron-ion collisions. We shall correct the electron mean free path to take into account the electron-neutral collision term (Banks, 1966). We define the electron mean free path as

$\frac{1}{\lambda_{e}}=\frac{1}{\lambda_{e e}}+\frac{1}{\lambda_{e i}}+\frac{1}{\lambda_{e n}}$,

or as a function of the electron-charged particle free path $\lambda_{e c}$ :

$\lambda_{e}=\frac{\lambda_{e c}}{1+\lambda_{e c} / \lambda_{e n}}$.

The electron-neutral collisions tend to reduce the electron mean free path, and in the limit of low neutral particle densities we recover the electron mean free path value of a fully ionized plasma (Banks, 1966). It is important to note that the differential equations for the perturbation functions $X_{E}$ and $X_{T}$ have not been modified, thus the departure of the velocity distribution function from the Maxwellian state is still caused by Coulomb interactions through the two Fokker-Planck collision operators for distant interactions.

In the ionosphere, a so-called polarization electric field $\boldsymbol{E}$ builds up such that the ions and electrons are constrained to drift as a single gas, which maintains bulk charge neutrality. $\boldsymbol{E}$ is determined by the current $\boldsymbol{J}$ and it

Table 1. The normalized transport coefficients as defined in Eqs. (4) to (7) calculated for different values of $x_{\max }$ and compared with the ones given by Spitzer and Härm $\left(x_{\max }=3.2\right)$

\begin{tabular}{llllll}
\hline$x_{\max }$ & 2.8 & 3.2 & Spitzer-Härm & 3.6 & 4.0 \\
\hline$\gamma_{E}$ & 0.5740 & 0.5811 & 0.5816 & 0.5826 & 0.5832 \\
$\gamma_{T}$ & 0.2507 & 0.2677 & 0.2727 & 0.2715 & 0.2718 \\
$\delta_{E}$ & 0.4436 & 0.4622 & 0.4652 & 0.4672 & 0.4698 \\
$\delta_{T}$ & 0.1877 & 0.2149 & 0.2252 & 0.2228 & 0.2237 \\
\hline
\end{tabular}

exists whenever there is a gradient in the electron density or in the temperature (Min et al., 1993). It is given by

$\boldsymbol{E}=\frac{\boldsymbol{J}}{\sigma_{e}}+\frac{\nabla p_{e}}{e n_{e}}-\frac{\tau_{e}}{\sigma_{e}} \nabla T_{e}$.

If the field-aligned current is attributed to the flow of the suprathermal electrons only then the $\boldsymbol{J} / \sigma_{e}$ term is small compared with the gradient terms and we get the following relation between the electric field $\boldsymbol{E}$ and the gradient of temperature $\nabla T_{e}$

$\boldsymbol{E}=\frac{\nabla p_{e}}{e n_{e}}-\frac{3 \gamma_{T} K_{b}}{2 \gamma_{E} e} \nabla T_{e}$.

Using Eqs. (1) to (7), this leads to the following relationship between the two Knudsen numbers $\epsilon_{E}$ and $\epsilon_{T}$

$4 \epsilon_{E} \gamma_{E}+3 \epsilon_{T} \gamma_{T}=0$.

In the rest of this paper we always consider the presence of such a polarization electric field. The two Knudsen numbers for the Spitzer-Härm distribution then always satisfy Eq. (13).

\section{The suprathermal velocity distribution}

The suprathermal velocity distribution $f_{s}$ we use is derived from the angular energy flux $\phi$ calculated by the electron transport model code along the Earth magnetic field described in Lilensten et al. (1989) and Lummerzheim and Lilensten (1994).

In the ionosphere, primary photoelectrons or precipitating electrons move along the magnetic field, produce heat and provoke processes such as excitation and ionization. In an ionization process, the incident electron mostly scattered forward is called the primary electron, while the extracted electron may be scattered in any direction and is called the secondary electron. This code calculates the energy flux of the electrons by solving the vertical kinetic transport equation. This equation simply expresses the fact that the variation of the steady-state electron flux with the scattering depth for a given altitude, energy and pitch angle, is the difference between whatever leaves that energy, altitude or angle slab and whatever enters it. The variations in energy or angle due to collisions are described through differential cross-sections. An additional energy loss arises from the heating of the ambient thermal electron gas due to hot electrons to thermal electrons interactions. This loss process is assumed to be a continuous energy loss of the hot electrons to the thermal electrons, without any deflection during the process.

We are using the angular energy flux calculated by this code as our input to calculate the velocity distribution. The electron velocity distribution is simply related to the angular energy flux by

$\phi(\boldsymbol{r}, E, \Omega, t)=\frac{v^{2}}{m_{e}} f_{s}(\boldsymbol{r}, E, \Omega, t) \quad \mathrm{eV}^{-1} \mathrm{~cm}^{-2} \mathrm{~s}^{-1}$

where $E=\frac{1}{2} m_{e} v^{2}$ and $\Omega$ is the solid angle. With the assumption that the angular energy flux is symmetric 
around the magnetic field, $f_{s}$ is a two-dimensional function of the energy $E$ or the velocity $v$ and of the pitch angle $\theta$ or the cosine of the pitch angle $\mu=\cos \theta$ to the magnetic field at a given altitude.

The angular energy flux $\phi$ is calculated over an energy grid of 215 points ranging from $E_{\min }=0.3 \mathrm{eV}$ to $E_{\max }=350 \mathrm{eV}$ and over a $\mu$-grid corresponding to the points of the double-Gauss quadrature integration rule (Stamnes et al., 1988). The number of points in the $\mu$-grid is often referred to as the number of streams. The doubleGauss quadrature refers to two Gauss quadratures applied separately on the upper and lower hemispheres. The main advantage of this double-Gauss scheme is that the quadrature points (in even orders) are distributed symmetrically around $|\mu|=0.5$ and clustered both towards $|\mu|=1$ and $\mu=0$, whereas in the single Gauss scheme they are clustered towards $|\mu|=1$. This clustering towards $\mu=0$ will give superior results near the boundaries where the functions to integrate vary rapidly or can even be discontinuous, i.e. around $\mu=0$.

The angular flux calculations we are using were obtained by running the code for 25 June 1994 at 14:00 UT over Tromsø assuming an $A_{p}$ index of 3 and a F10.7 index of 75 . The ionospheric parameters used as input to the code have been computed by the IRI 90 model (Bilitza, 1990).

Figures 2 and 3 show two examples of calculation of the distribution function for an eight-point angular quadrature. Figure 2 shows only the flux for one angle, the flux at this height is nearly isotropic and one could not separate the flux. From a height of about $200 \mathrm{~km}$ and above, the velocity distribution starts to develop an anisotropy mostly in the direction of the magnetic field, i.e. for $|\mu| \simeq 1$. This feature is clearly seen in Fig. 3: the two angular distributions in the lowest plate are for nearly parallel and anti-parallel directions to the magnetic field and they clearly present differences in intensity, while in the highest plate (angular distributions for the directions nearly perpendicular to the magnetic field), the two curves cannot be separated.

An interesting function which illustrates the regions in phase space where the heat flux is predominantly carried is the ratio of the integrated heat flux up to velocity $v=x v_{e}$ and normalized to the total net heat flux $q_{s}$ (Gray and Kilkenny, 1980). We define in this way the function $\alpha\left(v / v_{e}\right)$

$\alpha(x)=\frac{m_{e}}{2 q_{s}} \int_{0}^{v} \int_{-1}^{1}\left|\boldsymbol{u}-\boldsymbol{u}_{s}\right|^{2}\left(u \mu-u_{s}\right) f_{s}(u, \mu) 2 \pi u^{2} d \mu d u$,

where $\boldsymbol{u}_{\boldsymbol{s}}$ is the mean drift velocity of the suprathermal velocity distribution. Note that with the symmetry around the magnetic field both the mean drift velocity $\boldsymbol{u}_{\boldsymbol{s}}$ and $\boldsymbol{q}_{\boldsymbol{s}}$ are vectors parallel to the magnetic field of component $u_{s}$ and $q_{s}$, respectively.

Figure 4 shows the values of the parameter $\alpha$ at different altitudes for a standard set of suprathermal distribution function calculated by the transport code for an eight-stream run. At high altitudes (see Fig. 4 at $246 \mathrm{~km}$ for example), the local skewness is more than the net skewness for velocity $v \sim 30 v_{e}$, which means that

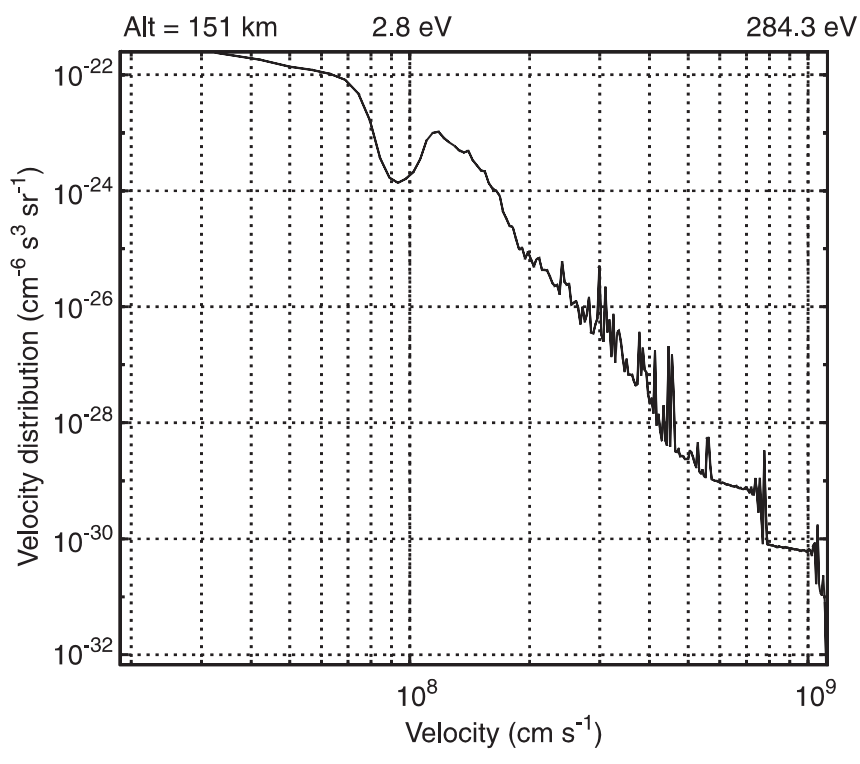

Fig. 2. The suprathermal angular velocity distribution function calculated by the transport code for an eight-stream calculation at $151 \mathrm{~km}$ and for a pitch angle of $86^{\circ}$ (i.e. $\mu=0.0694$ ). The distribution is nearly isotropic, and the data at the other pitch angles would not be distinguishable on the same plate

locally the distribution can have skewness of opposite sign compared to the total skewness of the distribution.

We now have a representation for the ambient and the suprathermal distributions, the next operation consists in the treatment of the transition region between the suprathermal and the ambient electrons. Sophisticated methods such as the numerical resolution of the nonlinear Boltzmann equation (Ashihara and Takayanagi, 1974; Jasperse, 1976), as well as full analytical treatment such as the one proposed by Krinberg (1973) have been studied to solve this problem. However, it has been shown later that a good approximation for the complete distribution function can be obtained by joining the two distribution functions at the energy for which the two distributions have equal intensities (Krinberg and Akatova, 1978; Stamnes and Rees, 1983). For simplicity we choose this method and in the rest of this paper the terminology truncated distribution refers to a distribution cut at the velocity where the ambient population equals the suprathermal population.

\section{Numerical two-dimensional plasma dispersion}

In linear theory the differential scattering cross-section $d^{2} \sigma / d \Omega d \omega$ per angular frequency and per solid angle for a multi-component, uniform, stationary, along the magnetic field and non-relativistic plasma with the collisions effects included through a BGK model is given by (Sheffield, 1975; Bjørnå and Trulsen, 1986; Ichimaru, 1992)

$\frac{d^{2} \sigma}{d \Omega d \omega}=\frac{1}{\sqrt{\pi}} n_{e} r_{0}^{2}|\boldsymbol{n} \times(\boldsymbol{n} \times \boldsymbol{p})|^{2} S(\boldsymbol{k}, \omega)$, 


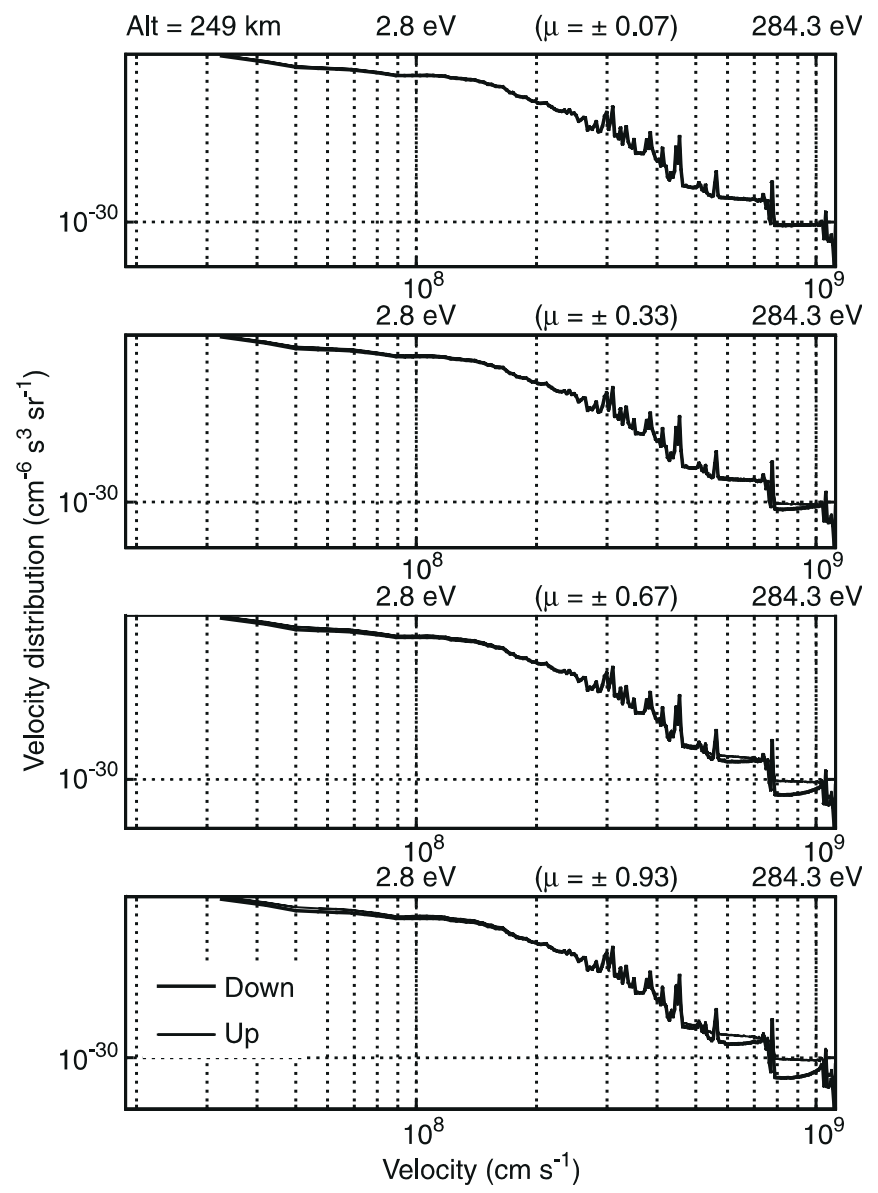

Fig. 3. The suprathermal angular velocity distribution function for the same eight-stream calculation at $249 \mathrm{~km}$. Each plate contains two curves corresponding to two angles symmetric around the direction perpendicular to the magnetic field, i.e the upward angular flux (thin solid line) and the downward one (thick solid line). The upper horizontal scale on each plate is energy expressed in $\mathrm{eV}$

where the spectral density function $S$ is defined as

$$
\begin{aligned}
& S(\boldsymbol{k}, \omega)=\left|1+\frac{C_{e}(\boldsymbol{k}, \omega)}{D(\boldsymbol{k}, \omega)}\right|^{2} \frac{\operatorname{Im} P_{e}(\boldsymbol{k}, \omega)-v_{e}\left|P_{e}(\boldsymbol{k}, \omega)\right|^{2}}{\sqrt{\pi}\left|X_{e}(\boldsymbol{k}, \omega)\right|^{2}} \\
& +\sum_{j} \frac{n_{j}}{n_{e}} z_{j}^{2}\left|\frac{C_{e}(\boldsymbol{k}, \omega)}{D(\boldsymbol{k}, \omega)}\right|^{2} \\
& \frac{\operatorname{Im} P_{j}(\boldsymbol{k}, \omega)-v_{j}\left|P_{j}(\boldsymbol{k}, \omega)\right|^{2}}{\sqrt{\pi}\left|X_{j}(\boldsymbol{k}, \omega)\right|^{2}},
\end{aligned}
$$

with

$$
\begin{aligned}
D(\boldsymbol{k}, \omega) & =1-\sum_{\alpha} C_{\alpha}(\boldsymbol{k}, \omega), \\
C_{\alpha}(\boldsymbol{k}, \omega) & =Z_{\alpha}(\boldsymbol{k}, \omega) / X_{\alpha}(\boldsymbol{k}, \omega), \\
X_{\alpha}(\boldsymbol{k}, \omega) & =1+i v_{\alpha} P_{\alpha}(\boldsymbol{k}, \omega), \\
Z_{\alpha}(\boldsymbol{k}, \omega) & =\sum_{k} Z_{\alpha, k}(\boldsymbol{k}, \omega), \\
Z_{\alpha, k}(\boldsymbol{k}, \omega) & =\frac{\omega_{\alpha, k}^{2}}{k^{2}} \int_{L} \frac{\boldsymbol{k} \cdot \nabla_{\boldsymbol{v}} \mathrm{f}_{\alpha, k}(\boldsymbol{v})}{\boldsymbol{k} \cdot \boldsymbol{v}-\omega-i v_{\alpha}} d^{3} \boldsymbol{v}
\end{aligned}
$$

$$
P_{\alpha}(\boldsymbol{k}, \omega)=\frac{1}{n_{\alpha}} \sum_{k} n_{\alpha, k} \int_{L} \frac{\mathrm{f}_{\alpha, k}(\boldsymbol{v})}{\boldsymbol{k} \cdot \boldsymbol{v}-\omega-i v_{\alpha}} d^{3} \boldsymbol{v} .
$$

$\mathrm{f}_{\alpha, k}=f_{\alpha, k} / n_{\alpha, k}$ denotes the velocity probability distribution function for the $k^{\text {th }}$ component of the particle species $\alpha$ ( $e$ for the electrons and $j$ for the ions). $v_{\alpha}$ is the collision frequency of the particle species $\alpha, r_{0}^{2}=e^{2} /\left(4 \pi \epsilon_{0} m_{e} c^{2}\right)$ is the electron radius, $\boldsymbol{n}$ is the unit vector pointing from the scattering volume towards the receiver and $\boldsymbol{p}$ is the unit polarization vector of the incident radiation; $\omega$ is the frequency shift between the transmitted radio wave $\omega_{0}$ and the received frequency $\omega_{r}, \boldsymbol{k}$ is the wave vector shift defined as the difference between the returned wave vector and the transmitted radio-wave vector $\boldsymbol{k}_{0}$.

$\omega=\omega_{r}-\omega_{0}$,

$\boldsymbol{k}=\frac{\omega_{r}}{c} \boldsymbol{n}-\boldsymbol{k}_{0}$.

$D$ and $Z_{\alpha}$ are respectively the dielectric function and the opposite of the susceptibility function for the particle species $\alpha$.

In order to calculate the dispersion relation, we need to calculate integrals of the $P$ and $Z$ types defined by

$Z(\boldsymbol{k}, \omega)=\frac{\omega_{e}^{2}}{k^{2}} \int_{\mathbf{L}} \frac{\boldsymbol{k} \cdot \nabla_{\boldsymbol{v}} f(\boldsymbol{v})}{\boldsymbol{k} \cdot \boldsymbol{v}-\omega-i v} d^{3} \boldsymbol{v}$

and

$P(\boldsymbol{k}, \omega)=\int_{\mathbf{L}} \frac{f(\boldsymbol{v})}{\boldsymbol{k} \cdot \boldsymbol{v}-\omega-i v} d^{3} \boldsymbol{v}$,

for velocity probability distribution $f$ defined in a cylindrical coordinate system along the magnetic field (which is the same direction as the temperature gradient), and when the scattered wave vector $\boldsymbol{k}$ is aligned to the local magnetic field line.

When $v=0$, one can note by applying the Plemelj formula that the imaginary part of $P$ is proportional to 

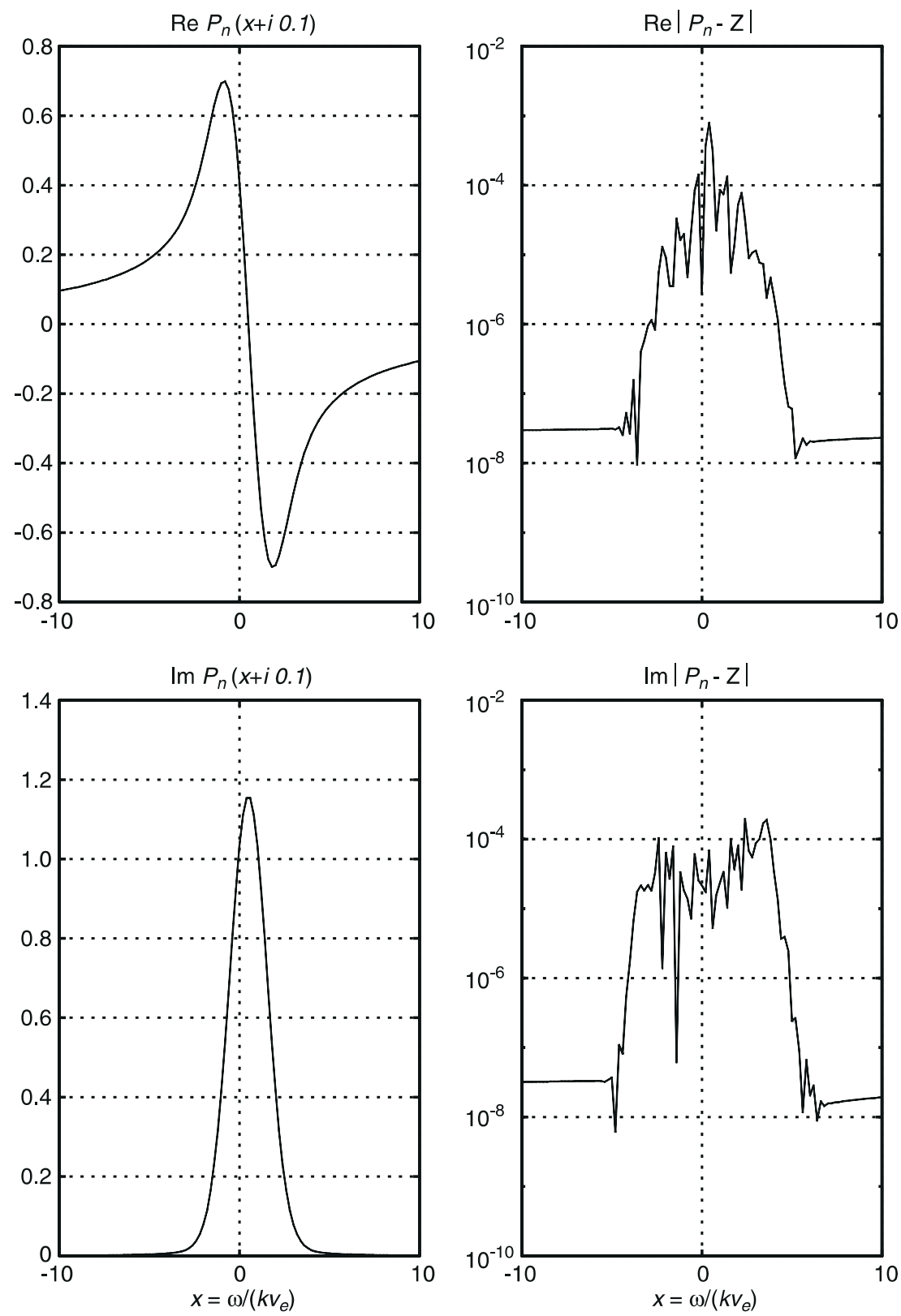

Fig. 5. On the left, the real and imaginary parts of the $P_{n}$ function given by Eq. (35) for complex argument such that the imaginary part $\eta=0.1$. On the right, their relative error with the real and imaginary parts of $\mathbf{Z}(z / \sqrt{2}) / \sqrt{2}$, where $\mathbf{Z}$ is the plasma dispersion function (Fried and Conte, 1961). The normalized Doppler shift of the Maxwellian distribution is $x_{\mathrm{d}}=0.5$

the reduced velocity distribution function $F_{\|}$along the direction of $\boldsymbol{k}$.

$F_{\|}\left(\frac{\omega}{k}\right)=\frac{k}{\pi} \operatorname{Im} P(\boldsymbol{k}, \omega)=\int f(\boldsymbol{v}) \delta(\boldsymbol{k} \cdot \boldsymbol{v}-\omega) \frac{d^{3} \boldsymbol{v}}{\pi}$.

When the collision frequencies are very small, we found that $P$ can be expressed in the form

$P(\boldsymbol{k}, \omega) \simeq \frac{1}{k v_{e}} P_{n}\left(\frac{\omega}{k v_{e}}\right)$,

with

$P_{n}(y)=2 \pi\left[\sum_{i=-n / 2}^{n / 2} w_{i} \int_{x_{1}}^{x_{2}} \frac{x^{2}}{\mu_{i}} \frac{v_{e}^{3} f\left(x v_{e}, \mu_{i}\right)}{x-y / \mu_{i}} d x\right.$

$i \neq 0$

$$
\left.+i \pi \sum_{i=1}^{n / 2} w_{i} \frac{|y|}{y} \frac{y^{2}}{\mu_{i}^{3}} v_{e}^{3} f\left(\frac{y v_{e}}{\mu_{i}}, \mu_{i}\right)\right],
$$

where $w_{i}$ and $\mu_{i}$ are respectively the weights and points of a $n$-points double-Gauss quadrature. In the same way, $Z$ can be formulated

$Z(\boldsymbol{k}, \omega) \simeq-\left(\frac{k_{s}}{k}\right)^{2} Z_{n}\left(\frac{\omega}{k v_{e}}\right)$

with

$Z_{n}(y)=-2 \pi\left[\sum_{i=-n / 2}^{n / 2} w_{i} \int_{x_{1}}^{x_{2}} \frac{x^{2}}{\mu_{i}} v_{e}^{3} \frac{\boldsymbol{n} \cdot \nabla_{\boldsymbol{v}} f\left(x v_{e}, \mu_{i}\right)}{x-y / \mu_{i}} d x\right.$

$i \neq 0$ 

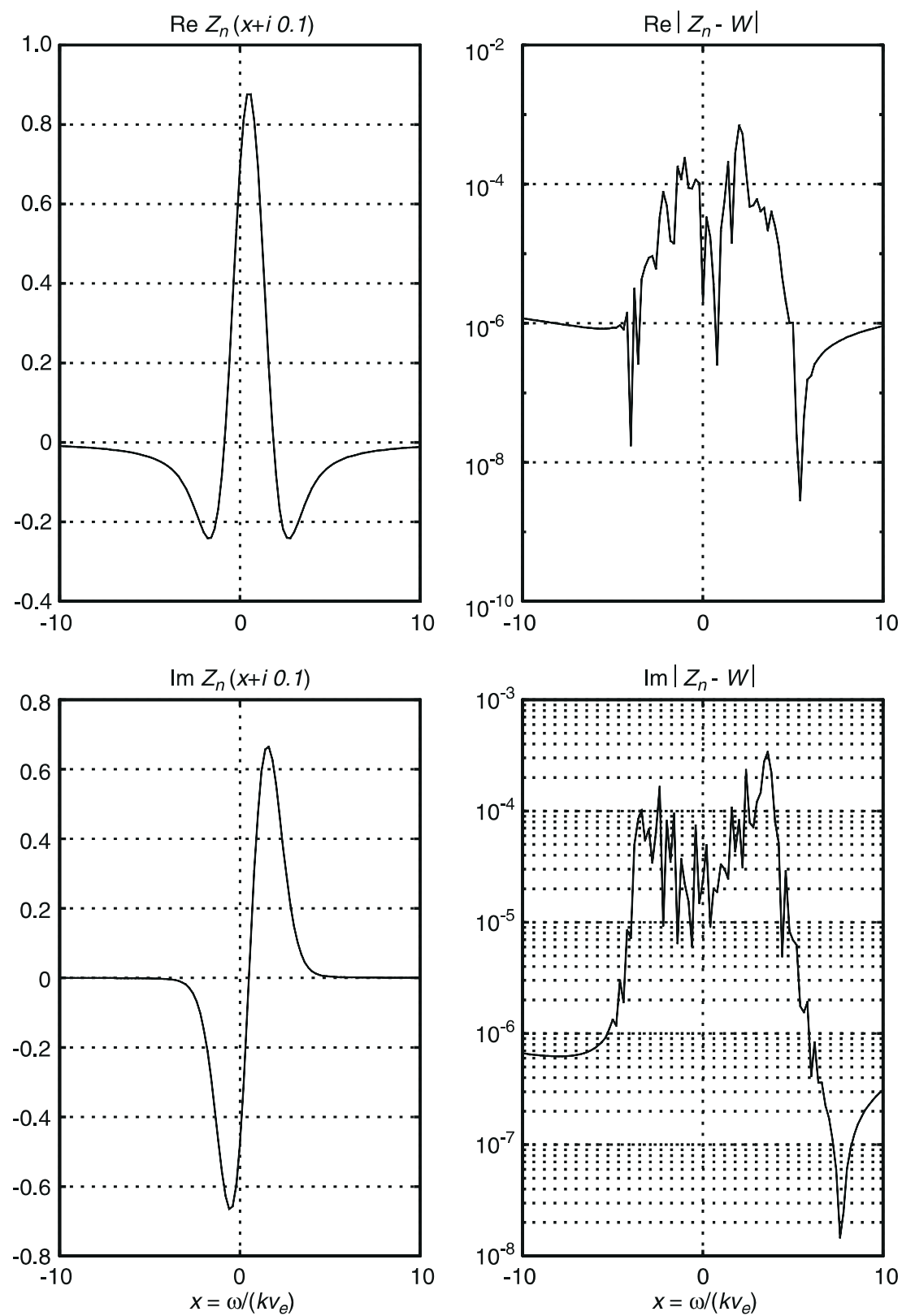

Fig. 6. On the left, the real and imaginary parts of the $Z_{n}$ function for same complex argument as in Fig. 5. On the right, their relative error with the real and imaginary parts of the $W$ function (Ichimaru, 1992). The normalized Doppler shift of the Maxwellian distribution is $x_{\mathrm{d}}=0.5$

$$
\left.+i \pi \sum_{i=1}^{n / 2} w_{i} \frac{|y|}{y} \frac{y^{2}}{\mu_{i}^{3}} v_{e}^{3} \boldsymbol{n} \cdot \nabla_{\boldsymbol{v}} f\left(\frac{y v_{e}}{\mu_{i}}, \mu_{i}\right)\right],
$$

where $\mathbf{n}=\boldsymbol{k} / k$ and

$\boldsymbol{n} \cdot \nabla_{\boldsymbol{v}} f(v, \mu)=\mu \frac{\partial f(v, \mu)}{\partial v}+\frac{1-\mu^{2}}{v} \frac{\partial f(v, \mu)}{\partial \mu}$.

When collisions are not negligible, the $P_{n}$ and $Z_{n}$ functions are modified to the following expressions

$$
\begin{aligned}
P_{n}(y+i \eta)= & 2 \pi \sum_{i=-n / 2}^{n / 2} w_{i} \\
& \times \int_{x_{1}}^{x_{2}} \frac{x^{2}}{\mu_{i}} v_{e}^{3} \frac{f\left(x v_{e}, \mu_{i}\right)\left(x-y / \mu_{i}+i \eta\right)}{\left(x-y / \mu_{i}\right)^{2}+\eta^{2}} d x
\end{aligned}
$$

and

$$
\begin{aligned}
Z_{n}(y+i \eta)= & 2 \pi \sum_{i=-n / 2}^{n / 2} w_{i} \\
& \times \int_{x_{1}}^{x_{2}} \frac{x^{2}}{\mu_{i}} v_{e}^{3} \frac{\boldsymbol{n} \cdot \nabla_{\boldsymbol{v}} f\left(x v_{e}, \mu_{i}\right)\left(x-y / \mu_{i}+i \eta\right)}{\left(x-y / \mu_{i}\right)^{2}+\eta^{2}} d x
\end{aligned}
$$

The integral over the normalized velocity is either of Cauchy principal values type or integral of rational functions. Two different quadratures are used to calculate these integrals.

\subsection{Test of $P_{n}$ and $Z_{n}$ on a Maxwellian}

We performed tests on the numerical evaluation of the $P_{n}$ and $Z_{n}$ functions for a Doppler-shifted two-dimensional Maxwellian distribution. The result for the $Z_{n}$ 

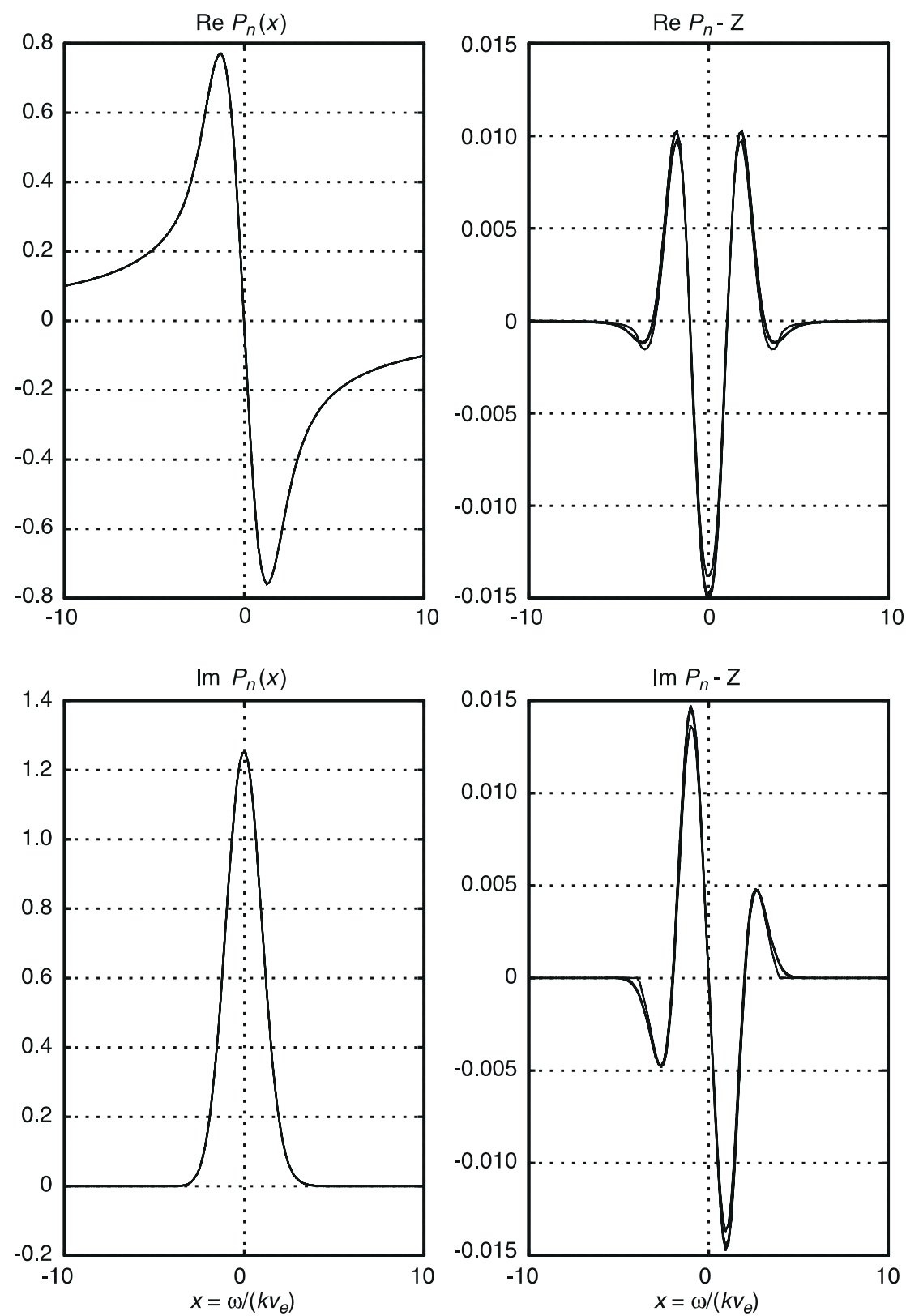

Fig. 7. On the left, the real and imaginary parts of $P_{n}$ for real argument $(\eta=0)$ and for Knudsen number $\epsilon_{T}=5 \cdot 10^{-2}$ and $\epsilon_{E}=-3 \epsilon_{T} \gamma_{T} / 4 \gamma_{E}$. On the right the difference between $P_{n}$ and $\mathbf{Z}(x / \sqrt{2}) / \sqrt{2}$ for the four different values of $x_{\max }$ of Table 1

function is compared with the $W$ function of a reduced Doppler-shifted Maxwellian (Ichimaru, 1992). The result for the $P_{n}$ function is compared with $\mathbf{Z}(x / \sqrt{2}) / \sqrt{2}$ where $\mathbf{Z}$ is the plasma dispersion function defined by Fried and Conte (1961).

The input for the code consists of a two-dimensional array filled with sampled data in both pitch angle and velocity. The velocity points are normalized to the mean drift velocity $v_{e}$. The parameters used for our test (Figs. 5 and 6) are, for the velocity space: 250 points ranging from 0 to $20 v_{e}$. It is much more than required and it is seen that the accuracy is not improved by increasing the sampling rate, nor by taking more points in the tail of the distribution function. On the other hand, the test shows that the precision is highly dependent on the number of points in the pitch angle quadrature for the calculation in the near thermal region, i.e. for $|v| \leq 4 v e$, but not too much for velocities $|v|>4 v e$.
In the thermal region, the accuracy is drastically improved by going from an eight-point double-Gauss quadrature (the relative error is about $10^{-1}$ ), to a 32 point quadrature where the relative error is better than $10^{-4}$. For larger velocities the accuracy is quite stable and is better than $10^{-7}$.

\subsection{Test of $P_{n}$ and $Z_{n}$ on the Spitzer-Härm distribution}

We also performed tests on the Spitzer-Härm distribution function. We looked at the influence of the upper boundary of integration $x_{\max }$ of the $X_{E}$ and $X_{T}$ functions when evaluating $P_{n}$ and $Z_{n}$. The values of $x_{\max }$ we used are the ones listed in Table 1.

For our test we used $\epsilon_{T}=5 \cdot 10^{-2}$, although the linear theory of heat conduction breaks down for such large values of $\epsilon_{T}$, that is these values give negative 
values of the velocity distribution function (Forslund, 1970). We used the same velocity grid as for the Maxwellian distribution while we increased the number of points in the pitch angle grid to 256 points. The results are shown in Figs. 7 and 8. One can see in the real part of the difference between $Z_{n}$ and $W$ in Fig.8, the artifact of the discontinuity of the distribution function at $x_{\max }$. This effect is larger for the lowest value $x_{\max }=2.8$ of the boundary i.e. $\omega / k v_{e}= \pm 2.8 \sqrt{2}$. For larger values of $x_{\max }$ the discontinuity of the thermal distribution is pushed down at higher velocities and is attenuated due to the Maxwellian behaviour at large velocities.

\subsection{Test of $P_{n}$ and $Z_{n}$ on the suprathermal distribution}

We used a 32-stream suprathermal calculation at an altitude of $202 \mathrm{~km}$ as input. The transport code calculation of the distribution function was then interpolated over a 1024 double-Gauss points. The $P_{n}$ and $Z_{n}$ functions were then computed using the distribution function evaluated on this denser $\mu$-grid. The suprathermal velocity distribution used are very much identical to the one presented in Fig. 3. When comparing with the $P_{n}$ and $Z_{n}$ functions of a Maxwellian or a Spitzer-Härm distribution, it is interesting to see how the characteristics of the distribution function are mapped on the $P_{n}$ and $Z_{n}$ shape. In order to integrate correctly the irregularities or 'spikes' corresponding to the discrete solar emission lines, we have to increase the order of the pitch angle quadrature up to 512 or even 1024 points. Increasing further the number of points in the $\mu$-grid space does not improve the results for large values of $\omega / k v_{e}$, i.e. above $\left|\omega / k v_{e}\right|>5$. On the other hand, for $\left|\omega / k v_{e}\right|<5$ the code is probably not so robust
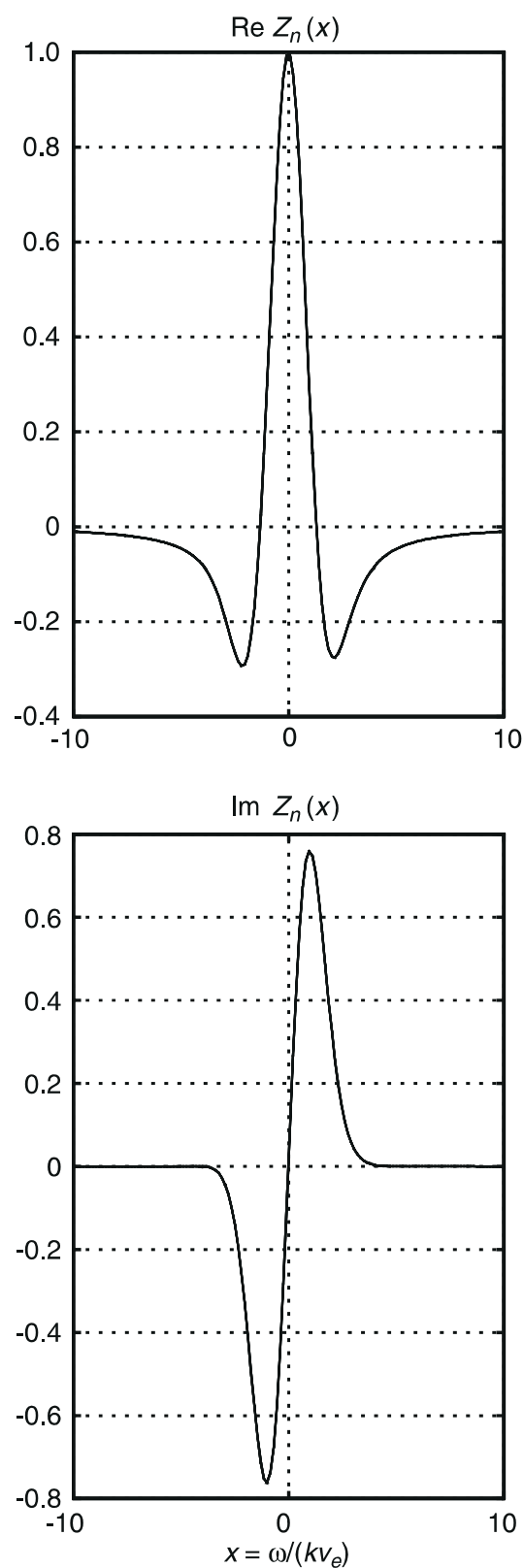
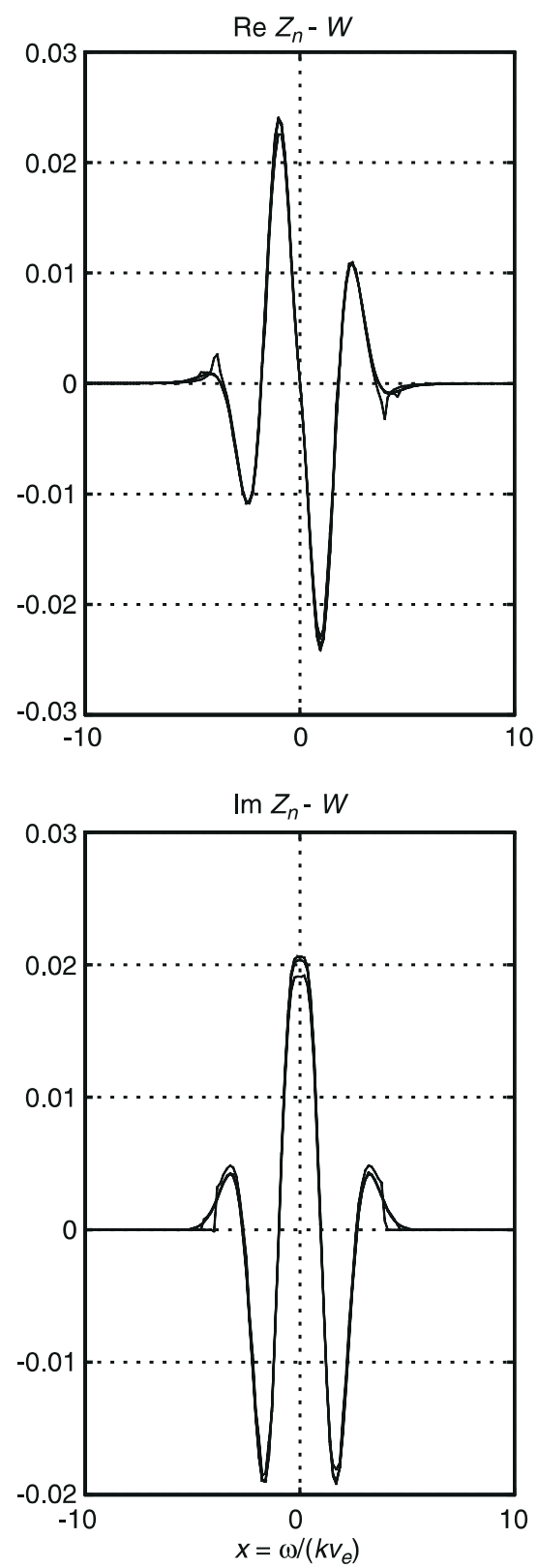

Fig. 8. On the left, the real and imaginary parts of $Z_{n}$ for real argument and for $\epsilon_{T}=5 \cdot 10^{-2}$ and $\epsilon_{E}=-3 \epsilon_{T} \gamma_{T} / 4 \gamma_{E}$. On the right the difference between $Z_{n}$ and $W$ for the four different values of $x_{\max }$ of Table 1 
to the spikes, as can be seen in the upper left plate in Fig. 9, and further developments need to be made.

There are several remarks to be made about the $P_{n}$ and $Z_{n}$ functions. First about the imaginary part of the $P_{n}$ function (lower left plate in Fig. 9) which is proportional to the reduced distribution function as is seen in Eq. (28). If the distribution were isotropic the flat part around zero should be equal on both sides of zero up to the value corresponding to the minimum energy of the suprathermal distribution. The effect of the anisotropy on the reduced velocity distribution function is to create a discontinuity at zero velocity and thus introduce a zero-order skewness. Secondly, on both the real and imaginary parts of the $Z_{n}$ functions (right plates in Fig. 9.), one can observe the signature of the distribution function itself. In particular, the typical $\mathrm{N}_{2}$ dip above 2 $\mathrm{eV}$ which corresponds to excitation of the vibrational levels in $\mathrm{N}_{2}$ (see Fig. 2) can clearly be identified around $\left|\omega / k v_{e}\right|=6.5$.

\section{Results}

We have used the two-dimensional code of the $P_{n}$ and $Z_{n}$ functions to calculate the frequency of the up- and downshifted Langmuir waves which are the highfrequency solutions of the plasma dispersion equation with the function $D(k, \omega)$ given in Eq. (18). We have performed these calculations for two different distributions, one that takes into account the deviation from the Maxwellian on the ambient part with the Spitzer-Harm distribution and the other one on the suprathermal part with the distribution calculated from the electron transport code.
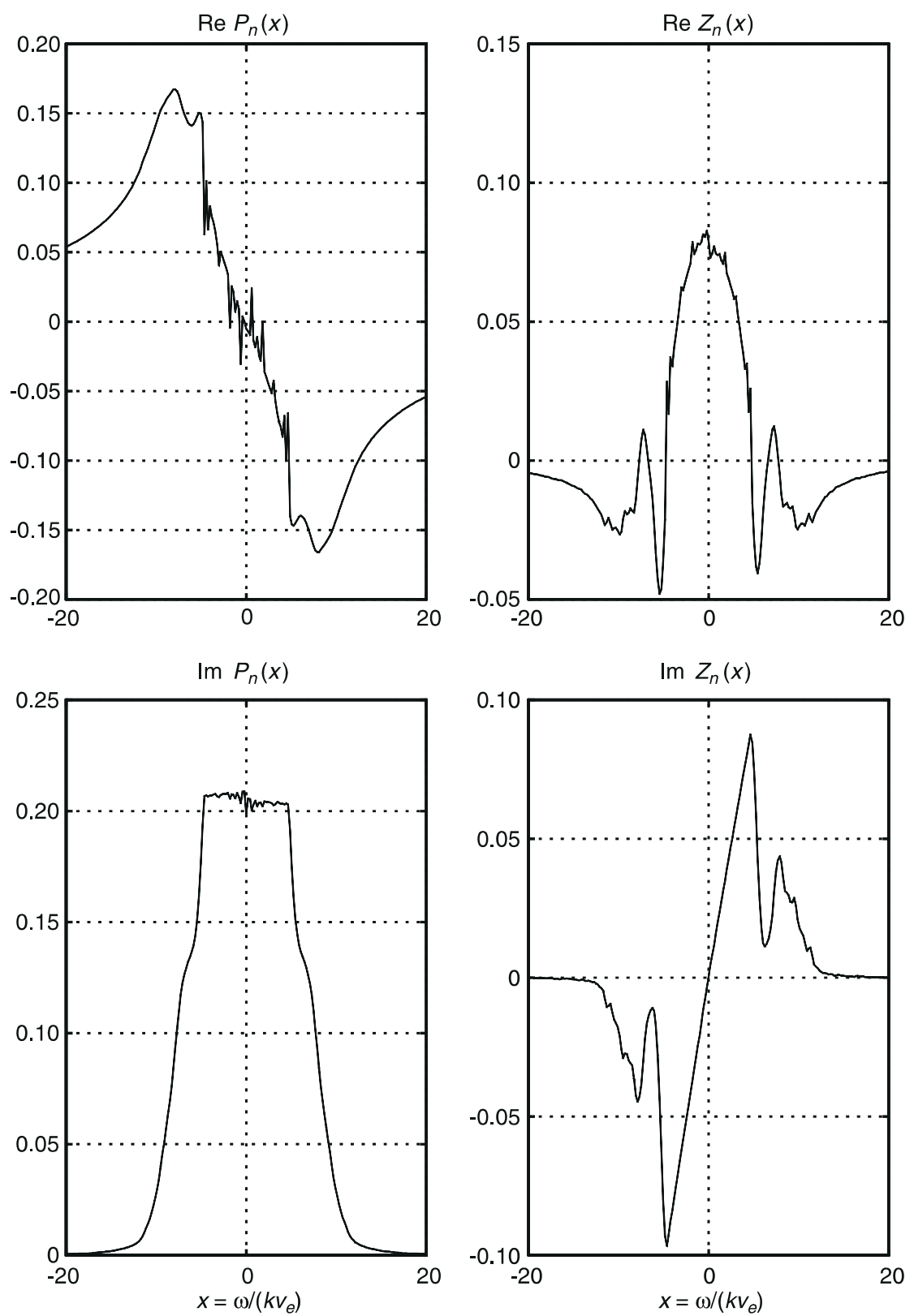

Fig. 9. On the left, the real and imaginary parts of $Z_{n}$ for real argument and on the right, the real and imaginary parts of $P_{n}$ for real argument of a suprathermal distribution at the altitude of $202 \mathrm{~km}$. These calculations were performed using a 32-stream calculation of the transport code and the distribution function was then recalculated over 1024 double-Gauss points in order to perform the calculations of $P_{n}$ and $Z_{n}$ over this $\mu$-grid 
We used the simulated data for 25 June 1994 at 14:00 UT over Tromsø assuming again an $A_{p}$ index of 3 and an F10.7 index of 75. The ionospheric parameters of the thermal part are shown in Fig. 10 and the velocity moments of the suprathermal distribution, as well as the moments of the Spitzer-Härm distribution, are shown in Fig. 11.

The lowest right plate in Fig. 10 shows the Knudsen number $\epsilon_{T}$ and $\epsilon_{E}$. The largest value is about $4.510^{-3}$. Such values are reasonable and allow the use of the linear theory of Spitzer-Härm. The corresponding polarization electric field $E$ of $\epsilon_{E}$ is also of the order of the expected value i.e. under $10^{-2} \mu \mathrm{V} \mathrm{m} \mathrm{m}^{-1}$.

Figure 11 shows the calculated suprathermal centred velocity moments up to the third order, i.e the heat flow, for both the raw distribution as calculated by the transport code and the truncated distribution we use in our calculations and which have been processed according to the strategy described at the end of Sect. 3. The lower right plate in Fig. 11 also shows the heat flow $q_{a}$ of the ambient Spitzer-Härm distribution function calculated numerically and the heat flow used by Kofman et al. (1993) which was originally given by Banks (1966)

$q_{\mathrm{B}}=-7.710^{5} T_{e}^{\frac{5}{2}} \nabla T_{e} \quad \mathrm{eV} \mathrm{cm}^{-2} \mathrm{~s}^{-1}$,

assuming a Coulomb logarithm $\log \Lambda=15$ and $\delta_{T}$ calculated by Spitzer and Härm (see Table 1). We note that the heat flow given by Eq. (36) has larger values by a factor up to 1.5 than the heat flow $q_{a}$ we calculated. The reason for this is that the approximation given by Eq. (36) is valid for a fully ionized gas only. We have taken into account the electron-neutron collisions in the mean free
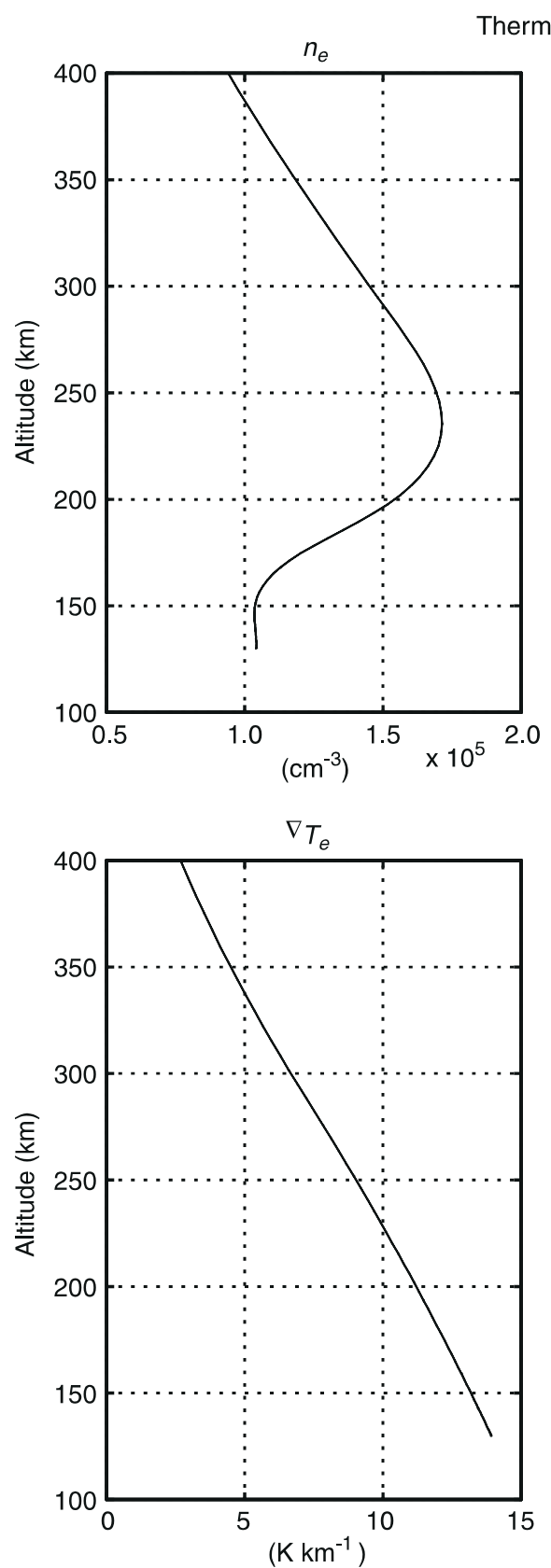
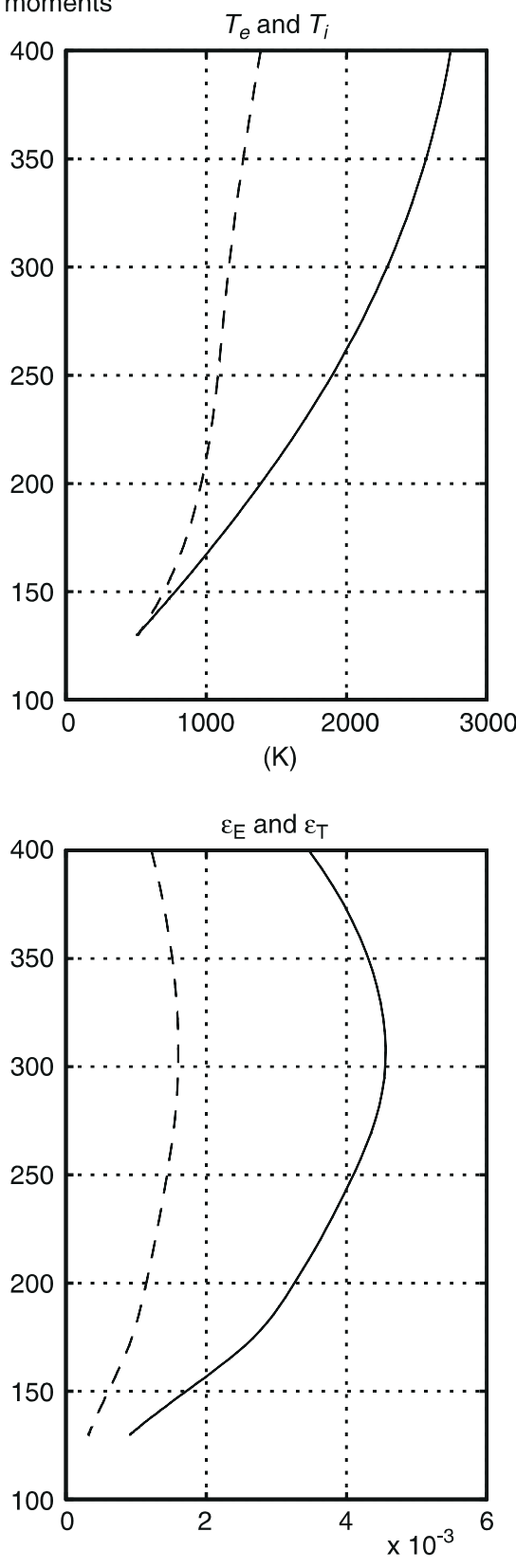

Fig. 10. The parameters for the ambient part of the distribution function, i.e. the electron density $n_{e}$, the electron and ion temperatures $T_{e}$ and $T_{i}$ (solid line and dashed line, respectively), the gradient of temperature $\nabla T_{e}$ and the two Knudsen numbers $\epsilon_{T}$ (solid line) and $\epsilon_{E}$ (dashed line) 

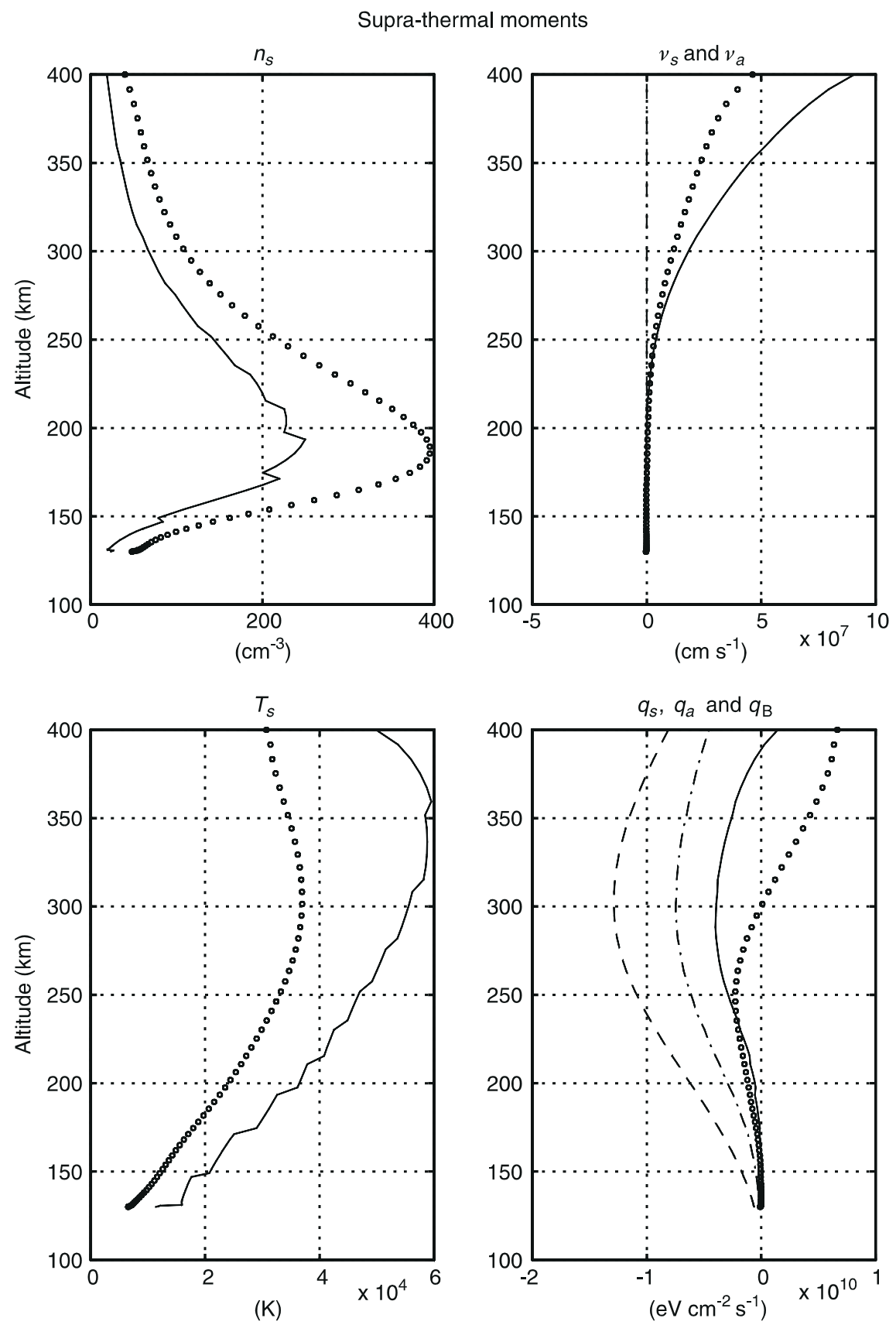

Fig. 11. The parameters of the suprathermal part of the distribution function and the two odd moments of the ambient (Spitzer-Härm) distribution. In all four plates, the moments of the raw suprathermal distribution function are represented by circles while the moments of the distribution we use for further calculations are represented by solid lines. In the upper right plate (mean drift velocity), the calculated mean Doppler velocity of the ambient distribution $v_{a}$ is represented by the dash-dot line and as expected is equal to zero (see Eqs. 11-13). On the lower right plate (heat flow), the calculated heat flow of the ambient distribution $q_{a}$ is represented by the dash-dot line and the dashed line corresponds to the heat flow $q_{\mathrm{B}}$ given by Eq. (36)

path (Eq. 10) and the effect is to decrease the two Knudsen numbers and thus the net heat flow (Banks, 1966).

Figures 12 and 13 show the frequencies of the upshifted Langmuir waves of the plasma lines and the frequency difference for the three EISCAT radars: VHF $(224 \mathrm{MHz})$, ESR $(500 \mathrm{MHz})$ and UHF $(931 \mathrm{MHz})$. Figure 12 shows the calculation for a deviation on the ambient part, i.e. the Spitzer-Härm distribution. The frequency asymmetry calculated is compared with the heat flow approximation of Eq. (9) of Kofman et al. (1993), Figure 13 shows the calculation in the presence of a suprathermal part and assuming that the ambient part is Maxwellian. The frequency asymmetry calculated is also compared with the results given by the heat flow approximation, assuming that the total distribution does not deviate dramatically from Maxwellian.
The best agreement between the full dispersion estimation and the heat flow approximation for the Spitzer-Härm distribution is for low-frequency radars like VHF radars. For these radars the phase velocity $v_{\phi}$ is between $12 v_{e}$ and $25 v_{e}$ as shown in Fig. 14. At such high velocities the moment approximation can be safely used, i.e. the classic expansion $(1-x)^{-1}=$ $1+x+x^{2}+\cdots+x^{n}$ is to be valid at the third order. For the UHF radar the phase velocity $v_{\phi}$ is between $3 v_{e}$ and $6 v_{e}$ (see Fig. 14) and the approximation breaks and we note a large deviation between the two calculations. This deviation can be observed on the real part of the difference between $Z_{n}$ and $W$ (upper right plate in Fig. 8) and has to be compared with the asymptotic behaviour in $\left(\omega / k v_{e}\right)^{-5}$ that we would get by subtracting $W$ to the heat flow approximation of Eq. (9) in 

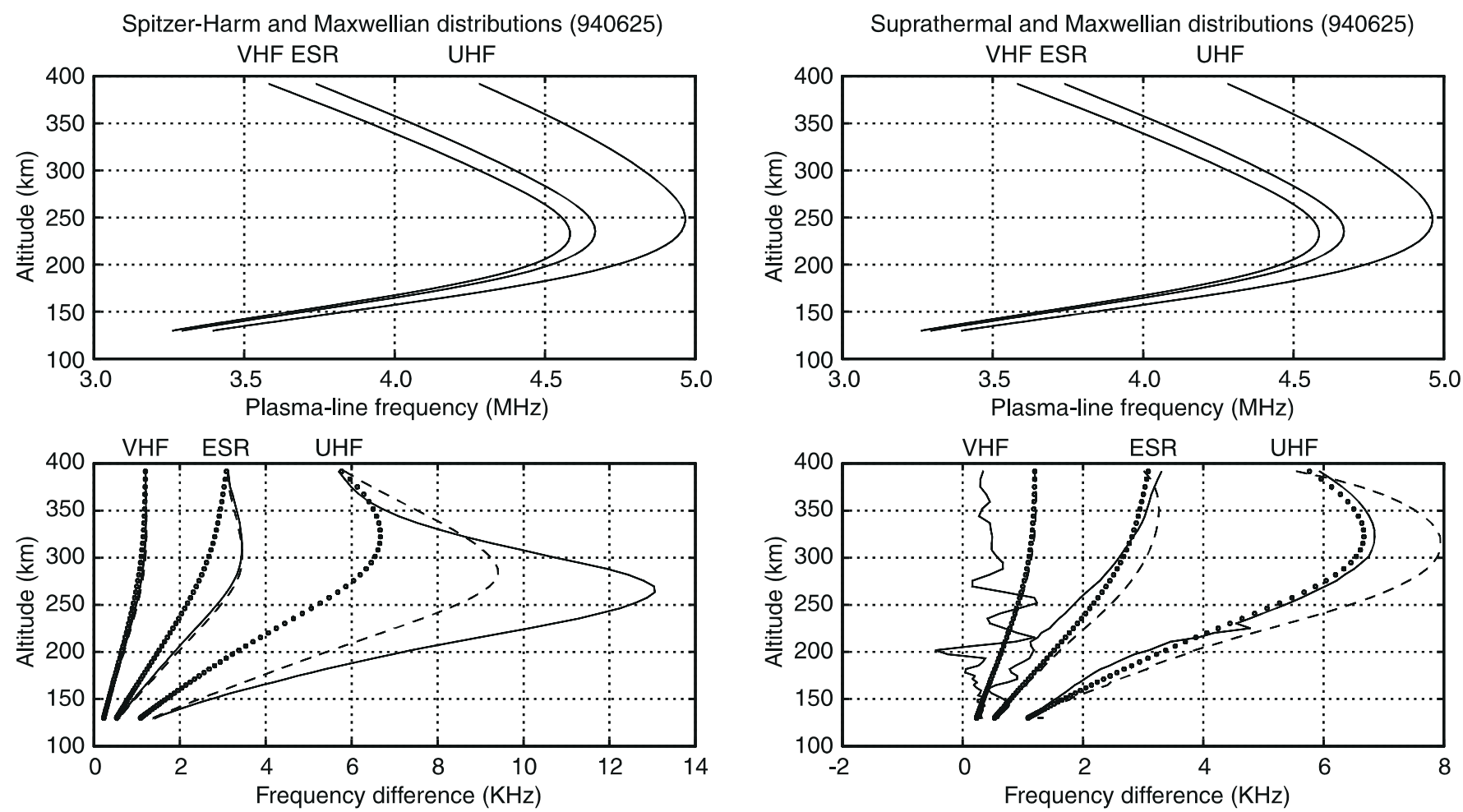

Fig. 12. The upper plate presents the calculated upshifted plasma frequency for the Spitzer-Härm distribution for the three different EISCAT radars. In the lower plate we present the frequency difference between up- and downshifted lines for the three radars. The Maxwellian approximation is shown with circles, the full twodimensional dispersion estimation is the solid line and the heat flow approximation (Kofman et al., 1993) is shown with the dashed line

Kofman et al. (1993), especially for values of $\omega / k v_{e}$ smaller than 5 .

Another remark is about the very large asymmetry observed around $250 \mathrm{~km}$, which is over $10 \mathrm{kHz}$ for the full dispersion calculation. We can see that due to the behaviour of the dispersion function at $4<\omega / k v_{e}<5$, we do not need large heat flow values to observe large asymmetry between the up- and downshifted plasma line frequencies. This is very satisfying in that we do not need to invoke larger heat flow values through processes such as the electron thermal runaway (Mishin and Hagfors, 1994; Nilsson et al., 1996) to explain the large deviation which were reported by Kofman et al. (1993), especially during 12 May 1992. On the contrary, our smaller heat flow values corrected for partially ionized plasma are in good agreement with the theory of Schunk and Walker (1970) and Banks (1966) and are able to create frequency asymmetry of the order of that observed by Kofman et al. (1993).

In the presence of a suprathermal distribution we can make the following remarks. For UHF radars, i.e. at phase velocity $v_{\phi}$ between $3 v_{e}$ and $6 v_{e}$, we note that the full dispersion calculation gives similar results as the Maxwellian approximation while the heat flow approximation gives larger deviation. In order to understand the small effect of the suprathermal distribution for highfrequency radars, we note that the real part of $Z_{n}$ of the thermal distribution (Fig. 6) has much larger amplitude

Fig. 13. Same plates as in Fig. 12. The compared distribution functions are a Maxwellian and a Maxwellian superposed with a suprathermal. The line codes are identical to the codes used in Fig. 12

than the one of the suprathermal distribution (Fig. 9) at the considered phase velocity. At large phase velocities $v_{\phi}$, i.e. for VHF radars, the thermal $Z_{n}$ is very small, whereas the one of the suprathermal is still not negligible. This is seen clearly when comparing the mean width of the real part of $Z_{n}$ in Fig. 6 and the real part of $Z_{n}$ in Fig. 9. Thus the effect of the suprathermal is important and should be taken into account. Another remark to be made is that if all the fine structures observed on the suprathermal $Z_{n}$ in Fig. 9 in the region $|\omega / k|<6 v_{e}$ are real and not artifacts of our calculations, they should map on the frequency asymmetry as it appears in Fig. 13.

\section{Conclusion}

We developed and tested a computer code to calculate the plasma dispersion function and the reduced distribution function for any arbitrary distribution function given in two dimensions: velocity and pitch angle. This code has been applied for two types of electron velocity distribution deviating from the Maxwellian distribution, one in the ambient part through a temperature gradient and the other one assuming the presence of a suprathermal electron population.

We used the code to estimate the frequency asymmetry between the up- and downshifted plasma lines which can be observed by incoherent-scatter radar technique. For high-frequency radars such as UHF radars we showed that the frequency asymmetry between the plasma lines is mostly due to a deviation from the Maxwellian in the ambient part of the electron 

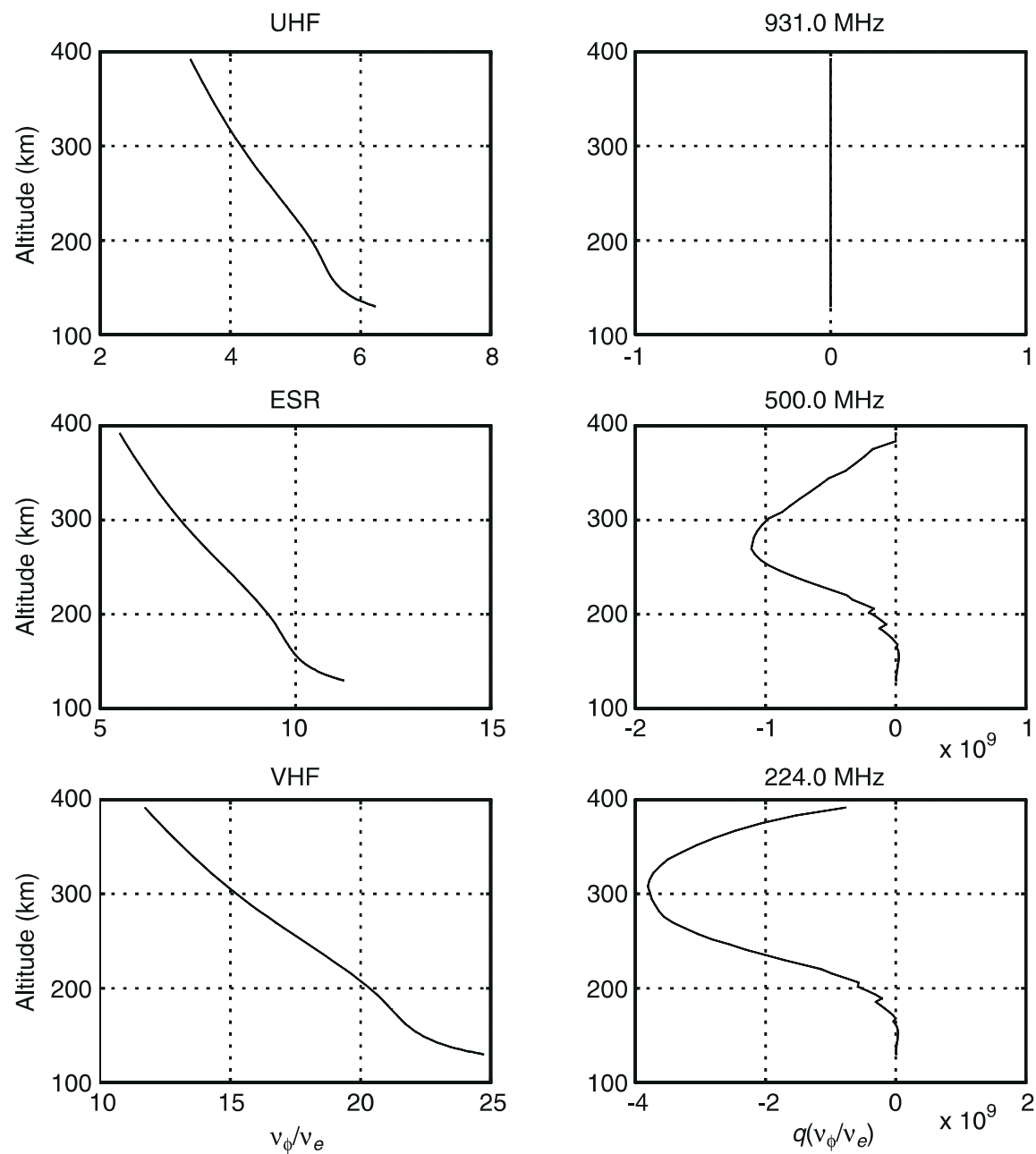

Fig. 14. In the left plates, the ratio $v_{\phi} / v_{e}$ where $v_{\phi}$ is the phase velocity of the Langmuir wave for the three different radars (from top to bottom UHF, ESR and VHF). In the right plates, the integrated heat flux up to the phase velocity $v_{\phi}$ distribution. On the other hand, for low-frequency radars such as VHF radars the Doppler frequency of the plasma lines is more influenced by the presence of a suprathermal electron population.

We also pointed out a discrepancy between the full estimation of the plasma dispersion function and the heat flow approximation for waves with phase velocity such that the moment expansion is not valid. The discrepancy is in the right direction and allows to explain large Doppler asymmetry of the plasma lines without need to increase the value of the heat flow. An analytic model of a distribution deviating from the Maxwellian distribution would be a very useful tool to study the difference between the exact calculation and the moment approximation of the plasma dispersion function.

Acknowledgements. The authors want to thank one of the referees for his very valuable remarks on the discussion of the results. This work was supported by the Norwegian Research Council (NFR). Topical Editor D. Alcaydé thanks J.-P. St.-Maurice and E.V. Mishin for their help in evaluating this paper.

\section{References}

Ashihara, O., and K. Takayanagi, Velocity distribution of ionospheric low-energy electrons, Planet. Space Sci., 22, 1201-1217, 1974.
Banks, P. M., Charged particle temperatures and electron thermal conductivity in the upper atmosphere, Ann. Geophys., 22, 577587, 1966

Bauer, P., K. D. Cole, and G. Lejeune, Field-aligned electric currents and their measurement by the incoherent backscatter technique, Planet. Space Sci., 24, 479-485, 1976.

Bilitza D., International reference ionosphere 1990. National Space Science Data center/ World Data Center-A for Rockets and Satellites, 1990.

Bjørnå, N., and J. Trulsen, Effect of a power law particle flux on the ionospheric incoherent scattering cross section, Phys. Scr., 33, 284-288, 1986.

Cohen, R. S., L. Spitzer Jr, and P. McRoutly, The electrical conductivity of an ionized gas, Phys. Rev., 80, 230-238, 1950.

Forslund, D. W., Instabilities associated with heat conduction in the solar wind and their consequences, J. Geophys. Res., 75, 1728, 1970 .

Fried, B. D., and S. D. Conte, The plasma dispersion function: the Hilbert transform of the Gaussian, Academic Press, New York, 1961.

Gombosi, T. I., and C. E. Rasmussen, Transport of gyrationdominated space plasmas of thermal origin. 1. Generalized transport equations, J. Geophys. Res., 96, 7759-7778, 1991.

Gray, D. R., and J. D. Kilkenny, The measurement of ion acoustic turbulence and reduced thermal conductivity caused by a large temperature gradient in a laser-heated plasma. Plasma Phys., 22, 81-111, 1980.

Ichimaru, S., Statistical plasma physics, Vol. 1, Addison-Wesley, Redwood City, 1992. 
Jasperse, J. R., Boltzmann-Fokker-Planck model for the electron distribution function in the Earth's ionosphere, Planet. Space Sci., 24, 33-40, 1976.

Kofman, W., J.-P. St-Maurice and A. P. van Eyken, Heat flow effect on the plasma line frequency, J. Geophys. Res., 98, 60796085,1993

Krinberg, I. A., Description of the photoelectron interaction with ambient electrons in the ionosphere, Planet. Space Sci., 21, 523$525,1973$.

Krinberg, I. A., and L. A. Akatova, Electron distribution function in the lower ionosphere and its relation with the rate of production and heating of electrons, Geomagn. Aeron., 18, 411-415, 1978

Lilensten, J., W. Kofman, J. Wisenberg, E. S. Oran and C. R. Devore, Ionization efficiency due to primary and secondary photoelectrons: a numerical model, Ann. Geophys., 7, 83-90, 1989.

Ljepojevic, N. N., and P. MacNeice, Heat flux in a non-Maxwellian plasma, Phys. Rev., A 40, 981-986, 1989.

Lummerzheim, D., and J. Lilensten, Electron transport and energy degradation in the ionosphere: evaluation of the numerical solution, comparison with laboratory experiments, auroral observations, Ann. Geophysicae, 12, 1039-1051, 1994.

Min, Q.-L., D. Lummerzheim, M. H. Rees, and K. Stamnes, Effects of a parallel electric field and the geomagnetic field in the topside ionosphere on auroral and photoelectron energy distributions, J. Geophys. Res., 98, 19223-19234, 1993.

Mishin, E. and T. Hagfors, On heat flow contribution to plasma line frequency in the F-region, J. Geophys. Res., 99, 6537-6539, 1994.
Nilsson, H., S. Kirkwood, and N. Bjørnå, Bistatic measurements of incoherent-scatter plasma lines, J. Atmos. Terr. Phys., 58, 175187, 1996.

Schunk, R. W., and J. C. G. Walker, Transport properties of the ionospheric electron gas, Planet. Space Sci., 18, 1535-1550, 1970.

Sheffield, J., Plasma scattering of electromagnetic radiation. Academic Press, New York, 1975.

Shkarofsky, I. P., Values of the transport coefficients in a plasma for any degree of, ionization based on a Maxwellian distribution, Can. J. Phys., 39, 1619-1668, 1961.

Showen, R. L., The spectral measurement of plasma lines, Radio Sci., 14, 503-508, 1979.

Spitzer, L. Jr and R. Härm, Transport phenomena in a completely ionized gas, Phys. Rev., 89, 977-981, 1953.

Stamnes, K., and M. H. Rees, Inelastic scattering effects on photoelectron spectra and ionospheric electron temperature, J. Geophys. Res., 88, 6301-6309, 1983.

Stamnes, K., S.-C. Tsay, W. Wiscombe, and K. Jayaweera, Numerically stable algorithm for discrete-ordinate-method radiative transfer in multiple scattering and emitting layered media, Appl Opt., 27, 2502-2509, 1988.

Vidal-Madjar, D., W. Kofman, and G. Lejeune, Mesures de la raie de plasma par diffusion incohérente à Nançay, et premiers résultats morphologiques, Ann. Geophys., 31, 227-234, 1975.

Woods L. C., An introduction to the kinetic theory of gases and magnetoplasmas. Oxford Science Publications, New York, 1993. 